\title{
PROCESS MODELING OF MESSAGE DISTRIBUTION IN SOCIAL NETWORKS BASED ON SOCIO-COMMUNICATIVE SOLITONS
}

\author{
Andrii Bomba 1), Nataliia Kunanets ${ }^{2)}$, Volodymyr Pasichnyk ${ }^{2)}$, Yuriy Turbal ${ }^{3)}$ \\ 1) Rivne State Humanitarian University, Ostafova str., 29, Rivne, Ukraine, abomba@ukr.net \\ ${ }^{2)}$ Lviv Polytechnic National University, 12 S. Bandery str., Lviv, 79000, Ukraine \\ 3) National University of Water and Environmental Engineering, Sobarna str.,11, Rivne, Ukraine, turbaly@gmail.com
}

Paper history:

Received 29 January 2018

Received in revised form 6 September 2018

Accepted 7 December 2018

Available online 31 December 2018

Keywords:

Social network;

graph of message flow;

excitation;

activity threshold;

Cauchy problem;

separated wave;

soliton;

front wave;

Boussinesq equation;

Korteweg-de Vries equation.

\begin{abstract}
The article proposes a new class of models for distributing messages in social networks based on socio- communicative solitons. This class of models enables to take into account the specific mechanisms for transmitting messages in the chains of the network graph, in which each of the vertices are individuals who, receiving a message, initially form their attitude towards it, and then decide on the further transmission of this message, provided that the corresponding potential of the interaction of two individuals exceeds a certain threshold level. The authors developed the original algorithm for calculating the time moments of message distribution in the corresponding chain, which comes to the solution of a series of Cauchy problems for systems of ordinary nonlinear differential equations. A special continualization procedure is formulated, which makes it possible to simplify substantially the resulting system of equations and replace a part of the equations by the Boussinesq or Korteweg-de Vries equations. The presence of soliton solutions to the above-mentioned equations provides grounds for considering socio-communicative solitons as an effective tool for modeling the processes of distributing messages in social networks and investigating the diverse influences on their dissemination processes.
\end{abstract}

Copyright (C) Research Institute for Intelligent Computer Systems, 2018. All rights reserved.

\section{INTRODUCTION}

The creation of the Internet and the launch of a wide range of social networks on its basis has led to a series of information and technological revolutionary transformations in society and has generated a number of new challenges both in the humanities and in the field of data analysis, mathematical modeling, physics, computer technology, etc. [2, 5-7]. New interdisciplinary research areas have emerged, such as Big Data, Data Mining, Cloud Computing, which are becoming increasingly widespread, particularly in solving many tasks related to the research and analysis of social networks. Functionally, the most important process that is implemented in social networks is the message dissemination that significantly affects both the attitudes and behavior of individuals and the formation of public opinion of groups and communities on certain issues. This is an effective motive for the creation of new models, which in a large number have appeared in recent years; authors considering models for distributing messages [9-10] and models for forming the opinions of both individuals and communities in general [13-16]. The conducted analysis confirms the validity of the assertion that all models should be divided according to the level of refinement into the corpuscular models, in which it is possible to identify an individual for certain multiple characteristics and generalized models describing the characteristics of groups of individuals or the community as a whole, and which allow us to form general ideas of processes of message distribution or public opinion formation (for example, the question can be about the number of individuals who spread rumors), etc.

The generalized models, for example, include the so-called epidemic models [2], the models of innovation diffusion $[18,20]$, the Delay-Kendall model [10], the model of message distribution in society [39], models based on the concept of message density [16-17]. Corpuscular models 
include a number of models that use cellular automata [18, 24], cascading models of various types [13], models of network autocorrelation [3], adaptive and imitation behavior model [12], "Game Name" model [18], quantum models, which are similar to Ising models $[26,27]$.

Probabilistic approaches, in particular, the Markov chains [3], are widely used in simulation of social and communication processes, in particular, various stochastic influences. The classes of tasks of forming and managing public opinion are important to solve problems that arise when it is necessary to change the opinions of individuals or target groups in a certain way due to the influence of certain agents [3, 39].

Along with a number of advantages, each of the above-mentioned model classes has its own limits of application, within which their adequacy is maximal. Therefore, in many cases, there is a need to develop new approaches to modeling that would combine the benefits of models of message dissemination processes and models of public opinion forming processes.

The aim of the work is to develop a new approach to modeling the process of message dissemination in social networks based on the procedures for synthesizing the existing patterns of message dissemination and processes of forming public opinion using the benefits of the latter.

The approach, proposed by the authors, is based on the combination of two basic essences. The first essence is the process of making an individual decision on the message distribution. This process resembles the process of transferring excitation to the nerve cell: if an input signal exceeds a certain threshold, a cell forms a certain signal at the output. Mathematical models of excitation transmission in the nerve fiber have been profoundly studied [30]. The fundamental foundation for this is the HodgkinHuxley model proposed in [31] for modeling the perturbation distribution in the axon of the squid and a series of simplified models, in particular, the FitzHugh-Nagumo model [32], Aliev-Panfilov model [34], Ziman model [35], Biktashev model $[36,37]$.The Hodgkin-Huxley model is presented by a system of differential equations in partial derivatives and has four variable membrane potential and permeability of the membrane for $\mathrm{K}+$, $\mathrm{Na}+$ ions and other types of ions. It should be noted that the use of the concept, mainly a threshold of individual activity in social networks was considered, particularly, in work [44].

The second essence is the well-known FermiPasta-Ulam model [45], which is used to simulate the processes of wave transmission in chains. It should be noted that the problem of the existence of a limited set of modes, which was experimentally discovered by Fermi, Pasta, and Ulam, only recently has been solved [43]. The application of this approach to social networks is also logical, since a set of individuals transmitting messages in a social network can also be submitted as a certain chain. If take into account the excitation threshold of the individual distributing messages, is possible to formulate certain modifications of the Fermi-PastaUlam model, for example, considering the cases when the system of balls analyzed in this model is located on brittle rods.

The basis for the creation by the authors of a new class of models for the message dissemination in social networks is, in particular, the work [1], in which it is proposed a model that can be attributed to discrete analogues of diffusion models of knowledge dissemination.

\section{THE ESSENCE OF THE PROPOSED NEW CLASS OF MODELS FOR MESSAGE DISTRIBUTION IN SOCIAL NETWORKS}

Let's consider the graph $G=(U, V)$ that describes the social network. Each individual is a member of the network given by the graph vertex and some vector $\bar{x}, \bar{x} \in U \subset R^{n}$. Components of this vector may include the geographical coordinates of the individual, the IP addresses of the computers from which the connection in the social network is done, etc. Each individual is in direct communication with a certain set of other individuals, for example, has several friends who form a particular community in the social network. The corresponding relationships will be described by the set of edges $V$. It should be noted that special objects, in particular, media that can distribute messages among a large number of participants at the same time may be the vertices of the graph $G$. Each individual can receive messages of $i$-type from others and transmit them. At the verbal level, it can be understood that some messages over time cause a certain reaction (excitation) of the individual. In the case when the excitation exceeds a certain threshold, the individual generates a message of the certain type that is passed on to its network partners.

An individual sends messages simultaneously to all partners, but they read them at different times (everyone can read it at any convenient time). If a particular individual is a source of a message, it can be constructed an oriented flow graph in which the arches indicate the possible direction of the message movement (Fig. 1). 


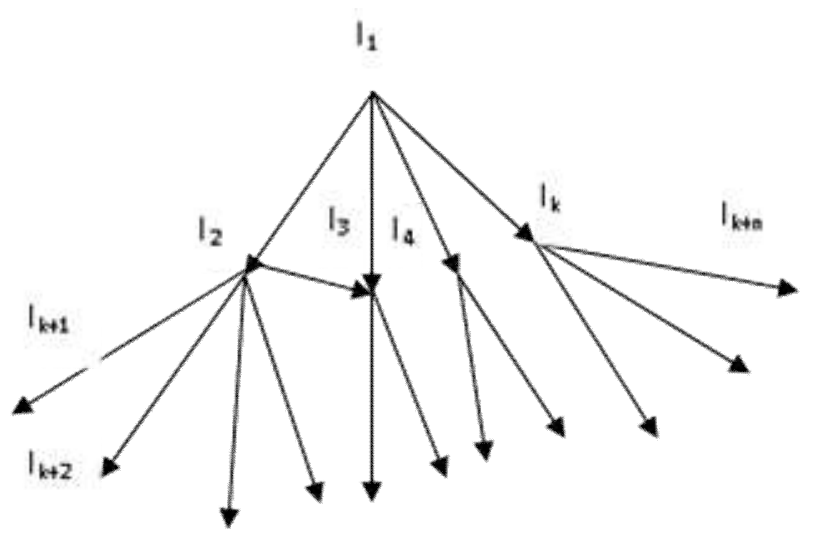

Figure 1 - Graph of message dissemination

The specificity of this representation is that each vertex is connected only to the vertices of the lower or the same layer. Such an oriented graph will be called the graph of message distribution or sociocommunicative graph $G(i)$. The process of distributing messages can be represented as ways going from the base graph top. In this case, the shortest way can be found from the base to all other vertices of the graph. If an individual receives a message, then he can generate a new message in the same context or forward the received message without changes, provided that its sociocommunicative potential exceeds a certain threshold. The message promotes an increase in sociocommunicative potential of an individual according to a certain law (equation describing excitation transfer along the axon), depending on the weight of the message. Receiving a re-message may also increase socio-communicative potential and cause further retransmitting of messages or participating in discussions and forums, etc.

Thus, it can be modeled an individual as a special "smart" neuron characterized by a special constantexcitation level, which changes continually with time and the mechanism for transmitting a message across a set of synapses, provided that the threshold level of excitation is exceeded (with the corresponding delay in signal transfer across the synapse).

When the message is transmitted from individuals to their partners, each one receives a message at a different time. In this context, for example, we may select the Erlang model, when the moments of receiving or transmitting messages form the random flow. At the same time, when an individual sends a message at the beginning of a certain time interval, then, after some interval, having no corresponding level of excitation, messages from an individual can continue to be received. Such an effect will be called phantom message distribution in the social network.

\section{ALGORITHM FOR MESSAGE TRANSMISSION IN AN ELEMENTARY SOCIAL NETWORK STRUCTURE}

Let's introduce a certain function $u(x, i, t)$ that is defined in a set $U$ and describes the excitation level of each individual (in the context of the message transmission), his deviation from the state of equilibrium caused by the message $i^{\prime} \in I$ at some point in time. It can be assumed that excitation extends from one individual to another through the message transmission of $i^{\prime} \in I$ type. If $u(x, i, t)>0$, then let's assume that an element $x$ will pass its excitation to other objects as a result of an increase in its excitation. If $u(x, i, t)<0$, then, the impact will be similar on the previous individual.

The mechanism for sending messages in the social network is described as follows. The excitation of an individual is determined by the excitation of his direct partners. Let $x_{1}, x_{2}$ be direct partners at the initial time $u\left(x_{1}, i, t\right)>0$, $u\left(x_{2}, i, t\right)=0$. It can be matched an edge $\left(x_{1}, x_{2}\right)$ with some function $\delta\left(x_{1}, x_{2}, i\right)$ that simulates the excitation transfer threshold caused by the message $i \in I$ from $x_{1}$ to $x_{2}$. In general, $\delta\left(x_{1}, x_{2}, i\right) \neq \delta\left(x_{1}, x_{2}, i\right)$. Let $f\left(u\left(x_{2}, i, t\right)-u\left(x_{1}, i, t\right)\right)$ be some interaction force between adjacent elements $x_{1}$ and $x_{2}$ depending on the level of their excitation. Let's assume that at the time when $f\left(0-u\left(x_{1}, i, t\right)\right)=\delta\left(x_{1}, x_{2}, i\right)$ the element $x_{1}$ affects the element $x_{2}$. The strength of influence at the initial moment of time $t \quad f\left(0-u\left(x_{1}, i, t\right)\right)$ will determine its excitation. At this time the value $u\left(x_{2}, i, t\right)$ begins to change. Consequently, taking into account the threshold, it can be introduced the interaction force:

$$
\begin{aligned}
& F\left(u\left(x_{2}, i, t\right)-u\left(x_{1}, i, t\right)\right)= \\
& =\left\{\begin{array}{l}
f\left(u\left(x_{2}, i, t\right)-u\left(x_{1}, i, t\right)\right), \text { if } \\
f\left(u\left(x_{2}, i, t\right)-u\left(x_{1}, i, t\right)\right)>\delta\left(x_{1}, x_{2}, i\right), \\
f\left(-u\left(x_{1}, i, t\right)\right), \text { if } \\
f\left(u\left(x_{2}, i, t\right)-u\left(x_{1}, i, t\right)\right) \leq \delta\left(x_{1}, x_{2}, i\right) .
\end{array}\right.
\end{aligned}
$$

In addition, let's suppose that at this moment of time the information exchange begins, the transmission of message $i^{\prime} \in I$ from an individual $x_{1}$ to an individual $x_{2}$, which results in increasing excitation of an individual $x_{2}$.

Let's introduce some analogue of the concept "weight" of an individual into the model in the 
context of the message transmission; the larger his weight is, the slower than individual responds to the change in the excitation of his neighbors. In this case, based on the analogue of the second law of Newton, it's possible to write the equation system of the dissemination of socio-communicative excitation:

$$
\begin{aligned}
& m_{k} u^{\prime \prime}\left(x_{k}, i, t\right)=F\left(u\left(x_{k+1}, i, t\right)-u\left(x_{k}, i, t\right)\right)- \\
& -F\left(u\left(x_{k}, i, t\right)-u\left(x_{k-1}, i, t\right)\right), \\
& k=\overline{1, n},\left(x_{k}, x_{k+1}\right) \in G(i)
\end{aligned}
$$

Without limitation of generality, let's assume that $u\left(x_{k}, i, t\right)=0, \quad u^{\prime}\left(x_{k}, i, t\right)=0, u\left(x_{0}, i, t\right)$ is a given function defining the initial perturbation.

It is possible to see that the process of information interaction can be detailed as follows. Let $\tau_{k}=\min \left\{t: f\left(0-u\left(x_{k-1}, i, t\right)\right)=\delta\left(x_{k-1}, x_{k}, i\right)\right\}$.

Then, at the time interval $\left[0, \tau_{1}\right]$ the sociocommunicative potential of an individual $x_{1}$ is unchanged and it is defined as the solution to the obvious Cauchy problem:

$$
\begin{gathered}
m_{1} u^{\prime \prime}\left(x_{1}, i, t\right)=0, \\
u\left(x_{1}, i, 0\right)=0, \\
u^{\prime}\left(x_{1}, i, 0\right)=0 .
\end{gathered}
$$

From the moment of time $\tau_{1}=\min \left\{t: f\left(0-u\left(x_{0}, i, t\right)\right)=\delta\left(x_{0}, x_{1}, i\right)\right\}$

the socio-communicative potential of an individual begins to change and its change in the interval of time $\left[\tau_{1}, \tau_{2}\right]$ will be determined as a solution to the problem:

$$
\begin{aligned}
& m_{1} u^{\prime \prime}\left(x_{1}, i, t\right)=f\left(0-u\left(x_{1}, i, t\right)\right)-f\left(u\left(x_{1}, i, t\right)-\right. \\
& \left.-u\left(x_{0}, i, t\right)\right), \tau_{1} \leq t \leq \tau_{2}
\end{aligned}
$$

Initial conditions are $u\left(x_{1}, i, \tau_{1}\right)=0, u^{\prime}\left(x_{1}, i, \tau_{1}\right)=0$.

Having solved the task (2), it can be determined the value $\tau_{2}$ by the formula:

$$
\tau_{2}=\min \left\{t: f\left(0-u\left(x_{1}, i, t\right)\right)=\delta\left(x_{1}, x_{2}, i\right)\right\} .
$$

Then the task for determining the change in socio-communicative potential of individuals $x_{1}$ and $x_{2}$ in the time interval $\left[\tau_{2}, \tau_{3}\right]$ can be presented as follows:

$$
\begin{aligned}
& \quad m_{1} u^{\prime \prime}\left(x_{1}, i, t\right)=f\left(u\left(x_{2}, i, t\right)-u\left(x_{1}, i, t\right)\right)- \\
& \quad-f\left(u\left(x_{1}, i, t\right)-u\left(x_{0}, i, t\right)\right), \\
& m_{2} u^{\prime \prime}\left(x_{2}, i, t\right)=f\left(0-u\left(x_{2}, i, t\right)\right)- \\
& -f\left(u\left(x_{2}, i, t\right)-u\left(x_{1}, i, t\right)\right),
\end{aligned}
$$

Initial values $u\left(x_{1}, i, \tau_{2}\right), u^{\prime}\left(x_{1}, i, \tau_{2}\right)$ are known from the previous equation, $u\left(x_{2}, i, \tau_{2}\right)=0$, $u^{\prime}\left(x_{2}, i, \tau_{2}\right)=0$.

Having solved the Cauchy problem (4), it can be determined:

$$
\tau_{3}=\min \left\{t: f\left(0-u\left(x_{2}, i, t\right)\right)=\delta\left(x_{2}, x_{3}, i\right)\right\}
$$

The following task is:

$$
\begin{gathered}
m_{1} u^{\prime \prime}\left(x_{1}, i, t\right)=f\left(u\left(x_{2}, i, t\right)-u\left(x_{1}, i, t\right)\right)- \\
-f\left(u\left(x_{1}, i, t\right)-u\left(x_{0}, i, t\right)\right), t \geq \tau_{3}, \\
m_{2} u^{\prime \prime}\left(x_{2}, i, t\right)=f\left(u\left(x_{3}, i, t\right)-u\left(x_{2}, i, t\right)\right)- \\
-f\left(u\left(x_{2}, i, t\right)-u\left(x_{1}, i, t\right)\right), t \geq \tau_{3}, \\
m_{3} u^{\prime \prime}\left(x_{3}, i, t\right)=f\left(0-u\left(x_{3}, i, t\right)\right)- \\
-f\left(u\left(x_{3}, i, t\right)-u\left(x_{2}, i, t\right)\right), \tau_{3} \leq t \leq \tau_{4} .
\end{gathered}
$$

Initial values

$u\left(x_{1}, i, \tau_{3}\right), u^{\prime}\left(x_{1}, i, \tau_{3}\right) u\left(x_{2}, i, \tau_{3}\right), u^{\prime}\left(x_{2}, i, \tau_{3}\right)$

are known from the previous task, $u\left(x_{3}, i, \tau_{3}\right)=0, u^{\prime}\left(x_{3}, i, \tau_{3}\right)=0$. Therefore, it can be determined:

$\tau_{4}=\min \left\{t: f\left(0-u\left(x_{3}, i, t\right)\right)=\delta\left(x_{3}, x_{4}, i\right)\right\}$.

Similarly, we may get the general Cauchy problem at $\tau_{k} \leq t \leq \tau_{k+1}$ :

$$
\left\{\begin{array}{l}
m_{1} u^{\prime \prime}\left(x_{1}, i, t\right)=f\left(u\left(x_{2}, i, t\right)-u\left(x_{1}, i, t\right)\right)- \\
-f\left(u\left(x_{1}, i, t\right)-u\left(x_{0}, i, t\right)\right), \\
m_{2} u^{\prime \prime}\left(x_{2}, i, t\right)=f\left(u\left(x_{3}, i, t\right)-u\left(x_{2}, i, t\right)\right)- \\
-f\left(u\left(x_{2}, i, t\right)-u\left(x_{1}, i, t\right)\right), \\
\ldots \\
m_{k-1} u^{\prime \prime}\left(x_{k-1}, i, t\right)=f\left(u\left(x_{k}, i, t\right)-u\left(x_{k-1}, i, t\right)\right)- \\
-f\left(u\left(x_{k-1}, i, t\right)-u\left(x_{k-2}, i, t\right)\right), \\
m_{k} u^{\prime \prime}\left(x_{k}, i, t\right)=f\left(0-u\left(x_{k}, i, t\right)\right)-f\left(u\left(x_{k}, i, t\right)-\right. \\
\left.-u\left(x_{k-1}, i, t\right)\right) .
\end{array}\right.
$$

Initial conditions: $u\left(x_{k}, i, \tau_{k}\right)=0, u^{\prime}\left(x_{k}, i, \tau_{k}\right)=0$, $u\left(x_{r-1}, i, \tau_{r}\right), u^{\prime}\left(x_{r-1}, i, \tau_{r}\right)$ are known, $r=\overline{1, k}$. $\tau_{k+1}=\min \left\{t: f\left(0-u\left(x_{k}, i, t\right)\right)=\delta\left(x_{k}, x_{k+1}, i\right)\right\}$.

$$
\text { If } f(x)=\alpha x+\beta x^{2} \text { or } f(x)=\alpha x+\beta x^{3} \text {, }
$$

then the system (6) can be rewritten as: 


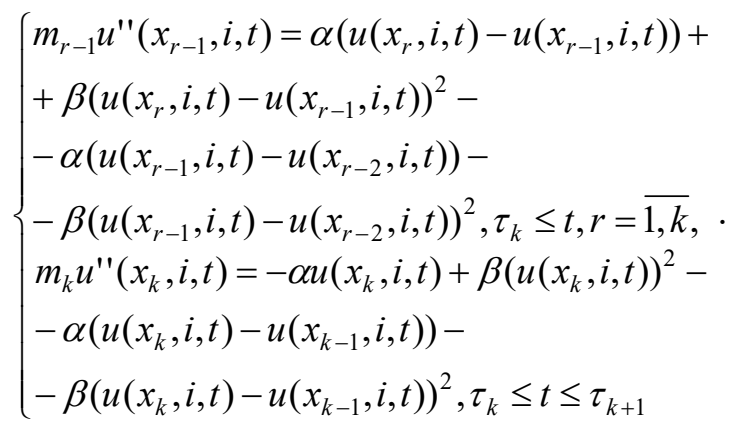

Hence,

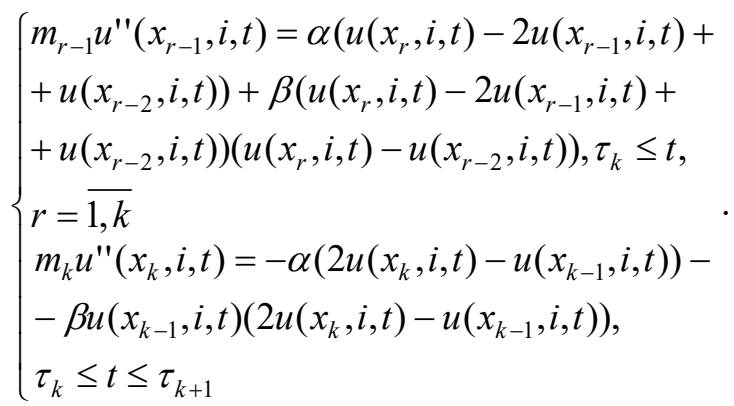

Thus, we get a series of Cauchy tasks, the solution of which allows us to find a sequence of time moments $\tau_{1}, \tau_{2}, \ldots$ that describe the beginning of an increase in the socio-communicative potential of the corresponding individuals in the chain or the transition to the next layer of the message flow. It is obvious that for any moment of time $t$, the value $\tau_{\max }(t)=\max \left\{\tau_{i}: \tau_{i} \leq t\right\}$ determines actually the front of the message dissemination wave.

\section{SIMPLIFICATION OF THE ALGORITHM ON THE BASIS OF THE CONTINUALIZATION PROCEDURE}

It is known to the experts that each of the tasks of the type (9) is quite cumbersome while solving. For the purpose of simplification, it is suggested to solve the following computational procedure. Let's replace the first $k-1$ of equations with some continuous analogues. Then we may get the equation of the type:

$$
\begin{aligned}
& m_{r-1} u^{\prime \prime}\left(x_{r-1}, i, t\right)=\alpha\left(u\left(x_{r}, i, t\right)-2 u\left(x_{r-1}, i, t\right)+\right. \\
& \left.+u\left(x_{r-2}, i, t\right)\right)+\beta\left(u\left(x_{r}, i, t\right)-2 u\left(x_{r-1}, i, t\right)+\right. \\
& \left.+u\left(x_{r-2}, i, t\right)\right)\left(u\left(x_{r}, i, t\right)-u\left(x_{r-2}, i, t\right)\right), \tau_{k} \leq t, \\
& r=\overline{1, k}
\end{aligned}
$$

Let's introduce a certain conditional distance between the individuals that is equal to $a$, and assume $(k-1) a=x$. Then $u\left(x_{k-1}, i, t\right)=u(x, i, t)$, $u\left(x_{k}, i, t\right)-u\left(x_{k-1}, i, t\right)=u(x+a, i, t)-u(x, i, t)=$

$=u_{x}(x, i, t) a+u_{x x}(x, i, t) \frac{a^{2}}{2}+u_{x x x}(x, i, t) \frac{a^{3}}{6}+$

$+u_{x x x x}(x, i, t) \frac{a^{4}}{24} \ldots$

$u\left(x_{k-1}, i, t\right)-u\left(x_{k-2}, i, t\right)=u(x, i, t)-$

$-u(x-a, i, t)=u_{x}(x, i, t) a-u_{x x}(x, i, t) \frac{a^{2}}{2}+$

$+u_{x x x}(x, i, t) \frac{a^{3}}{6}-u_{x x x x}(x, i, t) \frac{a^{4}}{24}+\ldots$

In such case we can rewrite the equation (10) in the following form:

$$
\begin{aligned}
& m_{k-1} u^{\prime \prime}{ }_{t}(x, i, t)=\alpha\left(u_{x}(x, i, t) a+u_{x x}(x, i, t) \frac{a^{2}}{2}+\right. \\
& \left.+u_{x x x}(x, i, t) \frac{a^{3}}{6}+u_{x x x x}(x, i, t) \frac{a^{4}}{24}\right)+ \\
& +\beta\left(u_{x}(x, i, t) a+u_{x x}(x, i, t) \frac{a^{2}}{2}+u_{x x x}(x, i, t) \frac{a^{3}}{6}+\right. \\
& \left.+u_{x x x x}(x, i, t) \frac{a^{4}}{24}\right)^{2}-\alpha\left(u_{x}(x, i, t) a-\right. \\
& -u_{x x}(x, i, t) \frac{a^{2}}{2}+u_{x x x}(x, i, t) \frac{a^{3}}{6}- \\
& \left.-u_{x x x x}(x, i, t) \frac{a^{4}}{24}\right)- \\
& -\beta\left(u_{x}(x, i, t) a-u_{x x}(x, i, t) \frac{a^{2}}{2}+u_{x x x}(x, i, t) \frac{a^{3}}{6}-\right. \\
& \left.-u_{x x x x}(x, i, t) \frac{a^{4}}{24}\right)^{2}, \tau_{k} \leq t
\end{aligned}
$$

$$
\begin{aligned}
& \text { Hence, it is } \\
& m_{k-1} u_{t}{ }_{t}(x, i, t)=\alpha\left(u_{x x}(x, i, t) a^{2}+\right. \\
& \left.+u_{x x x x}(x, i, t) \frac{a^{4}}{12}\right)+\beta u_{x x}(x, i, t) a^{2}\left(u_{x}(x, i, t) a+(\right. \\
& \left.+u_{x x x}(x, i, t) \frac{a^{3}}{6}\right), \tau_{k} \leq t
\end{aligned}
$$

If $u_{x}(x, i, t)=p(x, i, t)$, then from (12) we can get:

$$
\begin{aligned}
& m_{k-1} p^{\prime \prime}{ }_{t}(x, i, t)=\frac{\partial^{2}}{\partial x^{2}}\left(\alpha p(x, i, t) a^{2}+\right. \\
& \left.+\alpha p_{x x}(x, i, t) \frac{a^{4}}{12}+\beta a^{3} p^{2}(x, i, t) / 2\right)
\end{aligned}
$$

As a result of the transformation, we got the Boussinesq equation, which is known to obtain soliton solutions. Thus, instead of the system (8) let's consider the equation of the type: 
$m_{k} u^{\prime \prime}\left(x_{k}, i, t\right)=-\alpha\left(2 u\left(x_{k}, i, t\right)-u\left(x_{k-1}, i, t\right)\right)-$

$-\beta u\left(x_{k-1}, i, t\right)\left(2 u\left(x_{k}, i, t\right)-u\left(x_{k-1}, i, t\right)\right)$,

$\tau_{k} \leq t \leq \tau_{k+1}$,

where $\left.u\left(x_{k-1}, i, t\right)\right)$ is the solution of the Boussinesq equation. In this case, the described approach above is approximate, but it enables to simplify significantly the algorithm for calculating the values $\tau_{k}$ that simulate the front of the wave.

It should be noted that localized soliton-liked waves as solutions to systems (9) can be obtained directly without using the laborious procedure based on the T-representation method, proposed in paper [46].

Thus, it is possible to state about the existence of soliton solutions to model systems of differential equations. The concept of socio-communicative soliton is understood in a broader context, while considering a random localized wave of the message distribution in the social network (including the primary "shock" wave of message distribution), not emphasizing at the same time on some individual "soliton" properties, in particular, classical properties of the soliton interaction [44].

\section{SOME NUMERICAL MODELING RESULTS}

Let's try to simulate the processes of message dissemination in social networks on elementary chains based on the method proposed above using Mathcad 14.0. Assuming the threshold values of excitations of all individuals be equal to 0.001 , the initial perturbation is as follows: $u\left(x_{0}, i, t\right)=\exp \left(-(t-0.3)^{2} / 0.01\right)$. Then we can calculate the values $\tau_{1}$ :

$$
\tau_{1}=\min \left\{t: f\left(0-u\left(x_{0}, i, t\right)\right)=0.001\right\}=0.038 \text {. }
$$

Afterwards according to the proposed method, it is necessary to solve the Cauchy problems (3) - (5). The solution to the problem (3) is shown in Fig. 2.

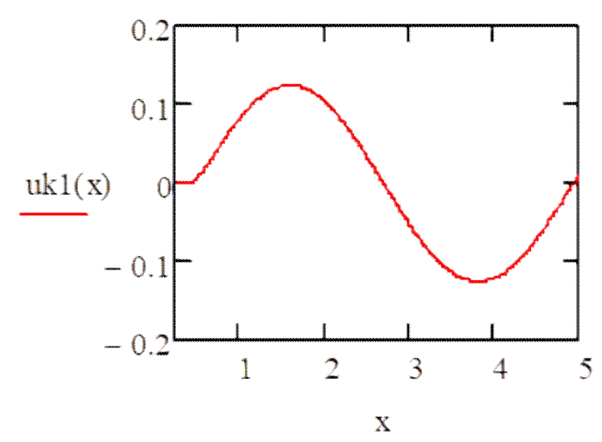

Figure 2 - Chart of the socio-communicative potential of the individual $x_{1}$
Let's formulate the problem (4) and solve it for the initial moment of time 0.038 . Changes in sociocommunicative potential of individuals $x_{1}$ and $x_{2}$ are shown in Fig. 3 and Fig. 4 correspondingly.

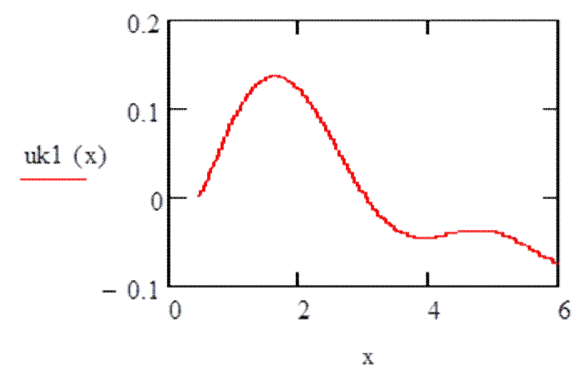

Figure 3 - Chart of the socio-communicative potential of the individual $x_{1}$ at $t \geq 0.038$

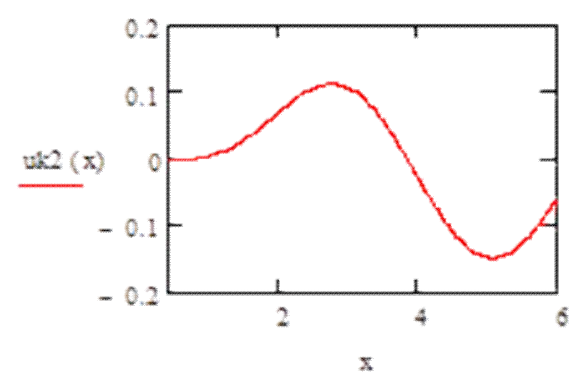

Fig. 4 Chart of the socio-communicative potential of the individual $x_{2}$ at $t \geq 0.038$

Then we can find $\tau_{2}$ :

$$
\tau_{2}=\min \left\{t: f\left(0-u\left(x_{1}, i, t\right)\right)=0.001\right\}=0.228 \text {. }
$$

Similarly, it's possible to solve problems (8) for $\mathrm{k}=4-7$. For $\mathrm{k}=7$, for example, a function $u\left(x_{k}, i, t\right)$ that is an integral part of the solution to the Cauchy problem (8) is shown in Fig. 5:

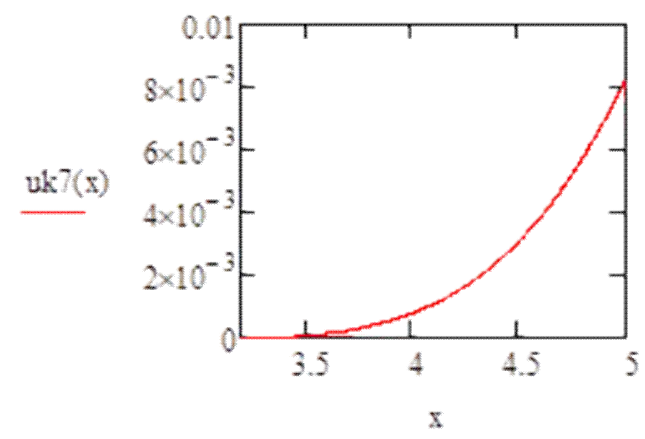

Figure 5 - Chart of the socio-communicative potential of the individual $x_{7}$

Thus, we may get a sequence of time moments in which each new individual in the chain of message distribution reaches socio-communicative excitation and becomes the distributor of the messages. Our example shows the corresponding time moments as follows: $0.038,0.228,0.666,1.338,1.636,2.356$, 3.195, 4.086. Let threshold socio-communicative 
perturbation of all individuals be the same and equal to 0.001 . If we modulate the eight levels of communication across the chain, then it's possible to get a function that illustrates the process of spreading the socio-communicative wave over time (Fig. 6). In Fig.6 the time is given along the axis of the abscissa and a spatial coordinate of spreading socio-communicative wave in the social network is shown along the axis of the ordinate.

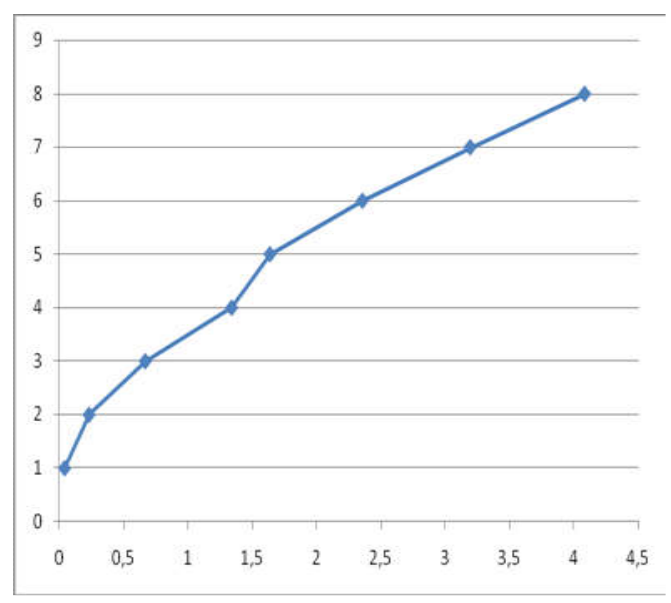

Figure 6 - Illustration of the process of spreading the front of the socio-communicative wave in time

Note: if times of activation $\tau_{1}, \tau_{2}, \ldots$ are known, then we can solve the system (6) or (7)-(8) and find the excitation levels $\delta\left(x_{2}, x_{3}, i\right)$ from the equation:

$$
\left.\left.\mid-\alpha u\left(x_{k}, i, \tau_{k+1}\right)\right)+\beta u^{2}\left(x_{k}, i, \tau_{k+1}\right)\right) \mid=\delta\left(x_{k}, x_{k+1}, i\right) .
$$

In this way we can estimate the parameters of our model for the real data. It is obvious that excitation levels depend on the type of information and can be considered as random variable.

As an example of the proposed approach above let's consider Harward Dataverse and Twitter user timelines belonging to Representatives in the House of the 115th U.S. Congress. They were collected from the Twitter API using Social Feed Manager [46]. This is a part of the series of timelines for the Senators Representatives tweets : RepMattGaetz on May 3, 2017, 10:49:48 a.m., RepRonEstes on May 4, 2017, 9:31:05 a.m., RepRyanZink on May 4, 2017, 9:32:37 a.m., RepRonEstes, on May 4, 2017, 9:40:36, RepMikeJohnson on May 5, 2017, 10:34:13 a.m., RepAnthonyBrown, on May 5, 2017, 10:37:51 a.m., RepRutherfordFL, on May 12, 2017, 10:38:02 a.m. So, we have normalized time series: 0,1 , $1.0015,1.0140,2.2196,2.2242,2.2258$. Using the proposed approach for parameters $u\left(x_{0}, i, t\right)=\exp \left(-(t-0.3)^{2}\right), a=10, b=20$, we get the corresponding excitation levels: 1.38 , $1.859 \mathrm{e}-5,2.224 \mathrm{e}-7,0.25,8.88 \mathrm{e}-5,1.427 \mathrm{e}-9$.

\section{CONCLUSION}

Thus, a new class of mathematical models for message distribution in social networks is constructed, which allows us to combine systematically the approaches used to model the processes of message dissemination and the processes of forming opinions of individuals and public opinion of the group in society. An important aspect of the proposed class of models is that the laborious procedure allows us to obtain the Boussinesq equation which provides soliton solutions. This result is not unexpected. After all, the separated waves in the resulting systems of differential equations of (7) type can also be directly obtained using the T-representation method. This clearly confirms once again the assumption made by the authors, that separate waves of the soliton type, play an important role in the processes of information exchange in social networks.

It should be noted that individuals in this model are actually considered as neurons that take qualified decisions regarding the further message distribution of a certain type. This approach, based on its essential grounds, is as close as possible to the real processes that occur in social networks, which enables to generate statements about the high level of adequacy of the proposed class of models.

The following mechanical analogy of the proposed class of models appears to be quite constructive when the balls with certain weights are connected by the springs and further fixed on the bare rods. In this case, the rods simulate the threshold level of an individual's excitation during transmission of messages. If the message is transmitted without taking into account the threshold of excitation, it is possible to get the classic FermiPasta-Ulam model. In comparison with the mechanical analogue the specificity of the proposed class of models is that in the social network the factors of energy loss due to the broken rods are not fixed. The latter circumstance is taken into account by the authors when constructing the corresponding systems of differential equations.

In this paper, the process of message dissemination is considered in detail only in one chain of a specially formed oriented graph. It is obvious that the holistic consideration of the entire graph enables to get a series of soliton effects that will be the subject of our further research.

\section{REFERENCES}

[1] A. Bomba, M. Nazaruk, N. Kunanets, V. Pasichnyk, "Constructing the diffusion-liked model of bicomponent knowledge potential distribution," International Journal of Computing, vol. 16, Issue 2, pp.74-81, 2017. 
[2] D. K. Horkovenko, "Overview of the models of information distribution in social networks," Young scientist, no. 8, pp. 23-28, 2017. (in Russian).

[3] H. A. Hubanov, D. A. Novikov, A. H. Chshartishvili, Social Networks: Modeling of Information Influence, Management and Confrontation, 2010, 228 p. (in Russian).

[4] H. A. Hubanov, "Review of online reputation / trust systems," Internet Conference on Governance Issues, 25 p., 2009. (in Russian).

[5] M. Cha, H. Haddadi, F. Benevenuto, K. P. Gummadi, "Measuring user influence in Twitter: the million follower fallacy," Proceedings of the ICWSM'10, 2010.

[6] M. Goetz, J. Leskovec, M. Mcglohon, C. Faloutsos, "Modeling blog dynamics," Proceedings of the ICWSM'09, 2009.

[7] J. Leskovec, L. Backstrom, J. Kleinberg, "Meme-tracking and the dynamics of the news cycle," Proceedings of the KDD'09, 2009.

[8] D. Liben-Nowell, J. Kleinberg, "Tracing information flow on a global scale using Internet chain-letter data," $P N A S$, vol. 105, issue 12, pp. 4633-4638, 2008.

[9] M. V. Nosova, L. I. Sennikova, "Modeling the information dissemination in decentralized network systems with irregular structure," New Information Technologies and Automated Systems, no. 17, pp. 8-15, 2014. (in Russian).

[10] D. J. Daley, D. G. Kendall, "Stochastic rumors," J. Inst. Math. Appl., vol. 142, pp. 42$55,1965$.

[11] D. Kempe, J. Kleinberg, E. Tardos, "Maximizing the spread of influence through a social network," Proceedings of the 9-th ACM SIGKDD International Conference on Knowledge Discovery and Data Mining, 2003, pp. 137-146.

[12] R. B. Myerson, Game Theory: Analysis of Conflict, London: Harvard Univ. Press, 1991.

[13] J. Goldenberg, B. Libai, E. Muller, "Talk of the Network: A Complex Systems Look at the Underlying Process of Word-of-Mouth," Marketing Letters, no. 2, pp. 11-34, 2001.

[14] H. W. Hethcote, "The mathematics of infectious diseases," SIAM Review, vol. 42, issue 4, pp. 599-653, 2000.

[15] R. Isea, R. Mayo-García, "Mathematical analysis of the spreading of a rumor among different subgroups of spreaders," Pure and Applied Mathematics Letters, vol. 2015, pp. 5054, 2015.

[16] S. K. Kuizheva, "About the mathematical tools of the research of social and economic systems," TERRA ECONOMICUS, vol. 12, no. 2, part 3, pp. 46-51, 2014. (in Russian).

[17] S. K. Kuizheva, "The Korteweg-de Vries equation and mathematical models of social and economic systems," Bulletin of the Adyghe State University. Series 4: naturalmathematical and technical sciences, no. 154, pp. 20-26, 2015. (in Russian).

[18] S. H. Lomakin, A. M. Phedotov, "Analysis of the model of information distribution in the cellular automata network," Bulletin of Novosibirsk State University. Series: Information Technology, pp. 86-97, 2014. (in Russian).

[19] V. A. Minaev, A. S. Ovchinskii, S. V. Skryl, S. N. Trostianskii, How to Control Mass Consciousness: Modern Models, Monograph, 2013, 200 p. (in Russian).

[20] E. Rogers, Diffusion of Innovations, 4 ed. N.Y.: Free Press, 1995.

[21] A. Baronchelli, M. Felici, E. Caglioti, V. Loreto, L. Steels, "Evolution of opinions on social networks in the presence of competing committed groups," J. Stat. Mech. URL: http://arxiv.org/ abs/1112.6414

[22] L. Dallsta, A. Baronchelli, A. Barrat, V. Loreto, "Non-equilibrium dynamics of language games on complex networks," URL: http://samarcanda.phys.uniroma1.it/vittorioloret o/publications/ language-dynamics/

[23] Q. Lu, G. Korniss, B. K. Szymanski, "Naming games in two-dimensional and small-worldconnected random geometric networks," URL: http://journals.aps.org/pre/abstract/10.1103/Phy sRevE.77.016111.

[24] A. Baronchelli, "Role of feedback and broadcasting in the naming game," Phys. Rev. E., vol. 83, Article 046103, 2011.

[25] A. I. Lobanov, "Models of cellular automata," Computer Studies and Modeling, no. 3, pp. 273-293, 2010. (in Russian).

[26] M. V. Altaiskii, N. E. Kaputkina, V. A. Krylov, "Quantum neural networks: current state and prospects of development," Physics of Elementary Particles and the Atomic Nucleus, vol. 45, 43 p., 2014. (in Russian).

[27] A. Cabello et al., "Quantum social networks," J. Math. Phys. A., vol. 45., pp. 285101, 2012.

[28] F. Beck, "Synaptic quantum tunnelling in brain activity," Neuroquantology, vol. 6, no. 2, pp. 140-151, 2008.

[29] Yu. E. Elkin, "The simplest models of excitable media".

[30] A. L. Hodgkin, A. F. Huxley, "A quantative description of membrane current and its application conduction and excitation in nerve," J. Physiol., no. 117, pp. 500-544, 1952.

[31] R. A. Fitzhugh, "Impulses and physiological 
states in theoretical model of nerve membrane," Biophys. J., no. 1, pp. 445-466, 1961.

[32] A. T. Winfree, "Varieties of spiral wave behaviour - an experimentalist's approach to the theory of excitable media," Chaos, no. 1, pp. 303-334, 1991.

[33] R. R. Aliev, A. V. Panfilov, "Asimple model of cardiac excitation," Chaos, Solitons \&Fractals, vol. 7, no. 3, pp. 293-301, 1996.

[34] E. C. Zeeman, Differential Equations for the Heartbeat and Nerve Impulses, Mathematical Institute, University of Warvick, Coventry, 1972.

[35] V. N. Biktashev, "Dissipation of excitation of wavefronts," Phys. Rev. Lett., no. 89(16), 2002.

[36] R. Suckley, V. N. Biktashev, "30 years on: a comparison of asymptotics of heart and nerve excitability".

[37] A. P. Mikhailov, A. P. Petrov, N. A. Marevtseva, I. V. Tretiakova, "Development of the model for the information dissemination in the society of 2014," Mathematical Modeling, vol. 26, issue 3, pp. 65-74, 2014. (in Russian).

[38] A. P. Mikhailov, K. V. Izmodenova, "About the optimal control of the propagation process of the formation," Mathematical Modeling, vol. 17 , no. 5, pp. 67-76, 2005. (in Russian).

[39] A. P. Mikhailov, N. A. Marevtseva, "Information fight models", Mathematical Modeling, vol. 23, no. 10, pp. 19-32, 2011. (in Russian).

[40] L. L. Delitsyn, Quantitative Models of the Spread of Innovations in the Field of Information and Telecommunication Technologies, 2009, 106 p. (in Russian).

[41] V. A. Shvedovskyi, P. A. Mykhailova, "Building an interaction model between electorates," Mathematical Modeling, vol. 20, no. 7, pp. 107-118, 2008. (in Russian).

[42] N. E. Friedkin, "Structural cohesion and equivalence explanations of social homogeneity," Sociological Methods and Research, no. 12, pp. 235-261, 1984.

[43] P. A. Prudkovskii, "The theory of nonlinear waves," W06 Waves in Chains.

[44] V. Yu. Novokshenov, Introduction to the Theory of solitons, Izhevsk, Institute for Computer Research, 2002, 96 p. (in Russian).

[45] A. Bomba, Y. Turbal, M. Turbal, "Method for studying the multi-solitone solutions of the Korteveg de-Vries type equations," Journal of Difference Equation, vol. 2, pp. 1-10, 2015.

[46] Yu. Turbal, "Necessary and sufficient conditions for the existence of solutions of motion equations for anisotropic elastic bodies I, n the form of solitary waves of the $\delta$-type - solitons," Problems of Applied Mathematics and Mathematical Modeling, no. 2012, pp. 7886, 2012. (in Russian).

[47] U.S. House Representatives (115th Congress) Official Twitter User Timelines, https://doi.org/10.7910/DVN/UIVHQR

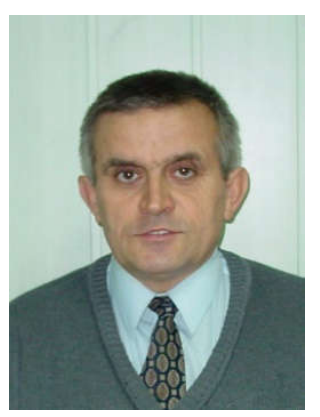

Andrii Bomba is $P h D$, Candidate of Physical and Mathematical Sciences, Professor, the Head of Department of Computer Science and Applied Mathematics at Rivne State Humanitarian University. $\mathrm{He}$ graduated from Lviv State Franko University, specialty "Mathematics"; he was a postgraduate student at the Institute of Mathematics of the USSR and doctoral candidate at V. M. Glushkov Institute of Cybernetics of NAS of Ukraine. $\mathrm{He}$ is a specialist in the field of mathematical modeling and computational methods.

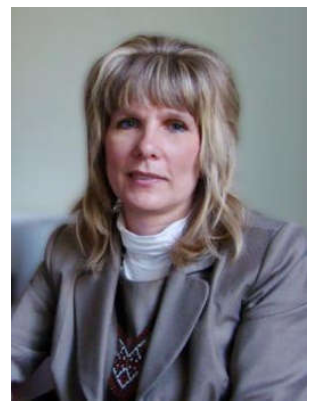

NataliiaKunanets is $P h D$ of Social Communications, Senior Fellow, Professor of the department of Information Systems and Networks, Deputy head of the department at Lviv Polytechnic National University.

She started her work way in 1981 at Lviv Stefanyk National Science Library. Since 2009 she has been working at Lviv Polytechnic National University. In 2007 she defended her thesis at Vernadsky National Library of Ukraine. In 2013 she defended her doctoral thesis for the degree of Doctor of Science of Social Communications. She conducts research on problems of social and communicative technology in projects "Smart City", information support of scientific research carrying out on the platform of escience and consolidation of information resources.

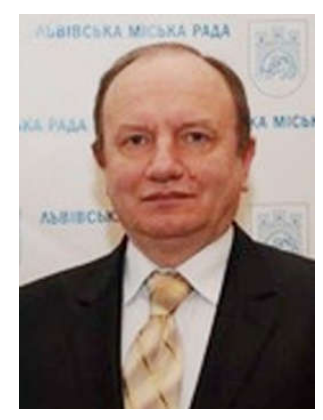

Volodymyr Pasichnyk is Professor, PhD, professor at the department of Information Systems and Networks, Institute of Computer Science and Information Technology, Lviv Polytechnic National University.

In 1978 he graduated with honour from the Faculty of Automation at Lviv Polytechnic National University, the specialty "Automated control systems" and with a qualification of system engineer. In 1984 he defended his thesis for the degree of Candidate of physical and mathematical 
Sciences in Specialized Academic Council of V. M. Glushkov Institute of Cybernetics of NAS of Ukraine. In 1994 he defended his thesis for the degree of doctor of technical sciences, specialty "Theoretical foundations of computer science". Since the early 90's, the scientific and professional interests are focused in the fields of creation and development of information systems for the real economy, production and non-production sphere.

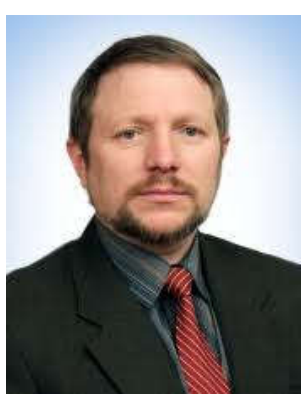

$$
\begin{aligned}
& \text { YuriyTurbal is PhD, } \\
& \text { Candidate of Physical and } \\
& \text { Mathematical Sciences, } \\
& \text { Professor of the Applied } \\
& \text { Mathematics Department at } \\
& \text { the National University of } \\
& \text { Water and Environmental } \\
& \text { Engineering. He holds a } \\
& \text { degree in Applied Mathematics } \\
& \text { at the Faculty of Cybernetics, }
\end{aligned}
$$

Taras Shevchenko National University of Kyiv. In 1996, he received his Doctor of Philosophy degree in Mathematics. In 2016 he received his Doctor of Engineering degree, specialty mathematical modeling and computational methods. Since the early 90's, the scientific and professional interests have been focused in the fields of mathematical modelling of the solitary wave propagation processes in continuous media and development of information systems for the solitary waves monitoring and forecasting. 